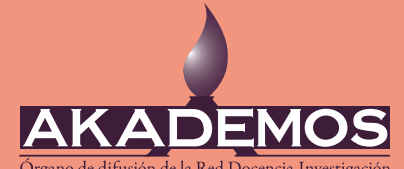

Órgano de difusión de la Red Docencia-Investigación

ISSN: $1995-4743$

Año 14 Vol. 2, n. 35 Julio-Diciembre 2020

\section{in art education}

Mario Zetino

Lic. en Letras. Investigador en Estudios literarios y Educación

Centro de Investigaciones en Ciencias y Humanidades (CICH)

Universidad Doctor José Matías Delgado

mesermenoz@ujmd.edu.sv

Marta Eugenia Valle Contreras, ${ }^{1}$ Manuel Alfaro Sifontes ${ }^{2}$

(Colaboradores)

\section{Resumen}

Se narra una investigación cuyo objetivo fue favorecer el desarrollo de capacidades y de competencias en un proceso de formación docente continua en educación artística con enfoque contemporáneo a través de la experiencia literaria. El estudio, de tipo experi- mental y cualitativo, se desarrolló por medio de la metodología de la investigación-acción, a través de un módulo de formación docente continua, que fue validado con la planta docente de una escuela rural. En la búsqueda de un proceso integral, tanto técnico como humano, el módulo empleó la metodología del enfoque educativo biocéntrico. Los resultados fueron un conocimiento vi-

1 Dra. en Artes y Educación. Investigadora en las áreas de Artes, Cultura y Educación en el Centro de Investigaciones en Ciencias y Humanidades (CICH) de la Universidad Doctor José Matías Delgado (UJMD). Coordinadora del Programa de Investigaciones para el Fomento de la Educación Artística, como parte del cual se desarrolla la investigación Proyecto de Formación Docente en Educación Artística (2017-2020), en el marco de la cual se realizó esta investigación. mevallec@ujmd.edu.sv

2 Mgtr. en Psicología Social-Comunitaria. Coordinador de Investigación y Proyección Social de la Facultad de Posgrados y Educación Continua en la Universidad Dr. José Matías Delgado. mgalfaros@ujmd.edu.sv 
vencial de las docentes participantes sobre la literatura, una experiencia de planificación por competencias para la asignatura de Educación Artística, una consolidación de la comunidad de trabajo de las docentes y una propuesta validada de módulo de formación continua en Educación Artística. Se concluye que una experiencia integral de la literatura, es decir que trascienda lo conceptual y se enfoque en lo experiencial, tiene el potencial para incidir positivamente en la práctica docente, a partir generar estados de bienestar y propiciar capacidades afectivas y creativas y el empoderamiento, tanto individual como grupal, en un equipo docente.

Palabras clave: educación artística, formación docente continua, educación literaria, paradigma biocéntrico, enfoque educativo biocéntrico

\section{Abstract}

The paper narrates an investigation which objective was to favor the development of capacities and competencies in a process of teacher training in art education through the literary experience. The study, of an experimental and qualitative type, was developed by means of the action research methodology, through a teacher training module, which was validated with the teaching staff of a rural school. In the search for an integral process, both technical and human, the module used the methodology of the Biocentric Education (proposed by Rolando Toro). The results were an experiential knowledge of the participating teachers about literature, an experience of planning by competencies for the subject of Art Education, a consolidation of the teacher's work community and a validated proposal for a teacher training module in Art Education. Conclusions are that an integral experience of literature, that is to say that transcends the conceptual and focuses on the experiential, has the potential to positively influence the teaching practice, from generating states of well-being and promoting affective and creative capacities and empowerment, both individual and group, in a teaching team.

Key words: art education, teacher education, literary education, biocentric paradigm, Biocentric Education

\section{Introducción}

La Agenda 2030 para el Desarrollo Sostenible de la ONU, en su objetivo 4, busca «Garantizar una educación inclusiva, equitativa y de calidad y promover oportunidades de aprendizaje durante toda la vida para todos». La formación docente en sus dos niveles, inicial y continua, es un elemento clave para el logro de este Objetivo de Desarrollo Sostenible (ODS), pues la calidad del docente es el factor más importante para la calidad educativa. Esta investigación busca contribuir a encontrar maneras de formar docentes con calidad. La investigación es sobre formación docente continua en educación artística y busca dar una respuesta a la pregunta: ¿cómo hacer posible que los aprendizajes de la formación docente continua se instalen y pasen a formar parte de las competencias de los docentes? 
Para dar una respuesta a esta pregunta, esta investigación, de tipo experimental y cualitativa, explora la experiencia literaria como un potenciador de los aprendizajes para docentes en procesos de formación continua. Esta propuesta se realiza con base en experiencias como las de Carlino \& Martínez (2009); Suárez (2014); Zubiri (2014); Labarthe \& Herrera (2016) y Neira (2017).

El contexto de la investigación es la urgencia de pasar del paradigma tradicional de la educación artística, que de manera muy amplia consiste en practicar técnicas artísticas, al paradigma contemporáneo: un desarrollo de las dimensiones artística, estética, ética y cultural de los estudiantes (Valle, 2011; Valle, 2016; Valle, Boulougne \& Zetino, 2017; Valle \& Zetino, 2019), pues este tipo de educación es la que puede ser el «fundamento para el desarrollo humano integral y sostenible» (Valle, 2016, p. 48).

En este contexto, la investigación se desarrolló como una investigación-acción, que surgió de una necesidad. En 2017, un centro escolar rural del departamento de La Libertad solicitó al Centro de Investigaciones en Ciencias y Humanidades (CICH) de la Universidad Dr. José Matías Delgado una intervención escolar: desarrollar una formación docente en educación artística para mejorar la calidad de la enseñanza aprendizaje de los estudiantes en dicha área.

A partir de esta solicitud, se inició la investigación Proyecto de Formación Docente en Educación Artística (2017-2019), la cual, por medio de la metodología de la investigación-acción (Perlo \& Sagastizabal, 2002; Blanchard, 2014; Chiva-Bartoll, Capella \& Pallarès, 2018), diseñó y validó junto con la planta docente del centro escolar una formación docente en Educación Artística de cuatro módulos, para contribuir a mejorar la calidad de la asignatura, y que posteriormente pudiera ser implementada en formación docente continua en esta área. Para Chiva, Capella \& Pallarès (2018) la investigación-acción es «uno de los modelos de investigación más adecuados para fomentar la calidad de la enseñanza e impulsar la figura del profesional investigador, reflexivo y en continua formación» (p. 279). ${ }^{3}$ El objetivo de la propuesta fue la promoción de capacidades (Nussbaum, 2012) y el desarrollo de competencias educativas (Tobón, 2005; MINED, 2017), las cuales se tradujeran en capacidades instaladas disponibles para la comunidad educativa del centro escolar.

Como tercer módulo de la formación, se desarrolló el de literatura, que es el que se

3 En El Salvador, ya en 1987, Martín-Baró consideraba que «el tipo de investigación que se involucra en los procesos mismos de cambio educativo es el más necesario en los momentos actuales para nuestros países, [... ] porque permite atacar los dos aspectos señalados de deterioro del sistema escolar: su deterioro cualitativo y su pérdida de contacto con la realidad social» (2010, p. 93). Y agregaba: «El modelo de "investigación acción" [...] supone un excelente ejemplo de este tipo de investigación que considero pertinente para la realidad actual de El Salvador» (Ídem.). 
relata en este artículo, ${ }^{4}$ con el objetivo de proporcionar a las docentes herramientas de dicho campo que potenciaran y fortalecieran la instalación de las capacidades y competencias que buscaba desarrollar la formación. Es decir, que posibilitaran que las docentes se apropiaran de dichos elementos, las capacidades y competencias, y los desarrollaran de manera autónoma, tanto durante el transcurso de la formación como tras la finalización de esta.

El módulo se desarrolló en nueve talleres entre octubre de 2018 y abril de 2019. Se trabajó junto a las docentes para que estas pudieran desarrollar la competencia didáctica en educación artística (MINED, 2017, pp. 10-11), que incluye competencias específicas que requieren de la escritura, tales como: la planificación didáctica por competencias; la investigación, educativa y artística; y el registro de experiencias pedagógicas (MINED, 2017). Y junto a las competencias, se buscó promover que las docentes desarrollaran capacidades, en el sentido del enfoque de las capacidades de Nussbaum (2012), para de este modo lograr una formación docente integral. Para posibilitar dicha integralidad, la formación docente se apoyó en los planteamientos y la metodología del enfoque educativo biocéntrico (Pérez, 2018).

Se trabajó con un total de 9 personas: la planta docente completa del centro escolar (5 personas: 3 profesoras, incluyendo a la directora; la facilitadora del área de refuerzo escolar, que es una Técnico en Trabajo Social; y la psicóloga del centro escolar) y el grupo de apoyo a la escuela de una ONG (4 personas: una gestora, dos encargadas de la escuela de valores para padres y una artista visual española perteneciente a la ONG en España, que realizaba una estadía voluntaria para enseñar educación artística en el centro escolar). Esta integración del grupo de apoyo de la ONG a la formación se realizó con el objetivo de instalar capacidades comunes y propiciar que los diversos actores tuvieran una visión compartida de la propuesta pedagógica, posibilitando así la comprensión, el apoyo y la continuidad de la propuesta formativa en el centro escolar luego de la investigación.

El perfil de las docentes es el de docentes generalistas que deben impartir Educación Artística. Este sería el perfil más común del docente de Educación Artística en El Salvador, ante la ausencia generalizada de docentes especialistas en Educación Artística (Valle, 2016, pp. 42-43). Además, en el centro escolar existe poco acceso al recurso humano para atender a los estudiantes, pues este atiende parvularia y primaria con sólo tres docentes: la directora es también profesora de parvularia, y las docentes de primaria imparten clase en aulas multigrado: de primero a tercer grado una maestra, y de cuarto a sexto la otra. 


\section{Marco teórico conceptual}

\section{Explorar estrategias alternativas de formación docente}

Ospina (2016) expone que, en el contexto de América,

Los estudios señalan que [la capacitación como solución al problema de la formación docente continua] resulta insuficiente[,] por su foco estrecho en torno a la perspectiva de formación y porque no posibilita auténticas transformaciones en las prácticas educativas[,] al desconocer las realidades de los contextos y de las instituciones. (p. 118)

Esta investigación busca medios para superar esas limitaciones, medios para anclar los aprendizajes y posibilitar su continuidad y desarrollo autónomo después de la formación. Con ese propósito, se busca integrar la experiencia literaria a una formación docente continua en educación artística, en al menos dos sentidos: primero, como una experiencia estética y artística, que se sume a las experiencias y planteamientos propios de la educación artística contemporánea y pueda enriquecer una formación docente continua en dicho campo; y luego, como un campo transversal en la práctica docente: como un conjunto de experiencias y herramientas a las que se puede recurrir en la vida personal para enriquecerla, y que pueden ser utilizadas o derivadas hacia la práctica docente en general y hacia la práctica docente en educación artística en particular.
La discusión sobre qué es la experiencia estética se remonta, en Occidente, a pensadores como Platón. Sin tratar de ser exhaustivos sobre este tema, cuya respuesta se actualiza con las épocas, las circunstancias y los pensadores, en este trabajo conceptualizamos como experiencia literaria aquella que le sucede a la persona en una relación con el texto literario, ya sea como lector o como escritor. Dicha experiencia es una experiencia estética, y como tal implica un evento emocional y tiene un carácter creativo. Lo emocional se refiere a que en la experiencia literaria se siente, hay una experimentación excepcional de emociones, el placer estético, tanto si se está leyendo como si se está escribiendo. Y lo creativo, por su parte, quiere recordar que la experiencia literaria no es una experiencia pasiva, sino que en ella, tanto si lee como si se escribe, la persona crea de distintas maneras: significados, sentidos lingüísticos y existenciales, expresiones, imágenes, conceptos, estructuras, comprensiones, etc. Es decir, la experiencia literaria es tanto un acontecimiento, algo que nos sucede, como un acto creativo, algo que hacemos, nos demos cuenta o no de ello, tanto en la subjetividad (al leer y al escribir) como en el plano objetivo (al escribir); es una experiencia en la cual la persona despliega capacidades humanas universales que la conectan con la dimensión de lo trascendente. A partir de Suárez (2014), decimos que la experiencia literaria «logra tocarle a la persona las fibras más profundas de su ser y lo lleva a ser alguien nuevo»; es un acontecimiento que «tiene el poder de transformar, de hacer cambiar la forma de sentir, de pensar, de ser» (p. 218). 
Con este enfoque, encontramos fundamentos para nuestra investigación en las de Suárez (2014) y Zubiri (2104), que tienen elementos en común, ya que ambos investigadores se alejan de las tendencias positivistas y formalistas de enseñanza de la literatura, y proponen estrategias alternativas de acercamiento a esta. Suárez (2014) desarrolló una investigación cuyo objetivo fue «establecer las condiciones pedagógicas para que la lectura sea una experiencia estético-literaria dentro del aula», asumiendo, «Epistemológicamente [...] la literatura desde su condición estética, es decir atendiendo a su poder creativo, imaginativo y sensitivo», poniendo «el acento en la fuerza figurativa y trascendental del texto literario para una vivencia emocional altamente enriquecedora y liberadora» (p. 215). Por su parte, Zubiri, en una experiencia con estudiantes de profesorado, propone una estrategia que «cede la centralidad a la escritura creativa como medio para empoderar al alumnado», relacionando el empoderamiento con la concienciación de Paulo Freire (Zubiri, 2014, pp. 60-62). Con estas propuestas, se invierte el binomio tradicional lectura-escritura (primero leer, después escribir, y escribir sobre todo como análisis, como comentario, o como respuesta a un cuestionario), para crear una dinámica participativa y motivadora, que involucre al estudiante no sólo con los aspectos conceptuales de la literatura, sino, antes que nada, con los experienciales, que resultan tener relación consigo mismo.

Esta forma de entender la enseñanza de la literatura, basándola en la escritura, ha sido ampliamente explorada por autoras como
Cameron (1999; 2016) y DeSalvo (2000) en el campo de la escritura creativa. Además, en el campo de la educación, Silvia Castrillón se refiere de este modo al binomio escritura-lectura:

Hablamos mucho de la lectura y poco de la escritura y eso también es una postura ideológica... se promueve más la lectura que la escritura porque la escritura es una forma de emancipación; la lectura también, pero la escritura más. Dar la palabra, expresarse a través de la escritura, es un paso más allá en la emancipación, es tener un pensamiento más libre. (Castrillón citada en Martínez, 2010, p. 105)

Y específicamente sobre educación artística, Martínez (2010), en un artículo sobre formación docente continua en esta área, señala que «la lectura y la escritura debieran ocupar un lugar significativo» en la formación del profesorado de educación artística:

$[\ldots]$ hablamos $[\ldots]$ de la práctica de la escritura sobre el hacer artístico como una actividad creativa que parece haber cedido demasiado espacio en dicha formación. Hablamos de la ausencia de tradición, en el profesorado, de una práctica de la escritura como herramienta de reconocimiento del arte y del hacer artístico, como una competencia transversal al hacer artístico [...]. (p. 105)

\section{La escritura docente}

La escritura docente suele entenderse como un tipo de escritura meramente técnica: la escritura necesaria para realizar las diversas 
tareas de la práctica docente, tales como la planificación didáctica, la enseñanza aprendizaje y el reporte de las actividades realizadas. Sin embargo, la escritura de los docentes no sólo es, y no sólo puede ser, una escritura profesional, técnica, sino que también posee, y en una gran proporción, un componente personal, emocional. Este componente se puede ver reflejado en textos como el registro de experiencias docentes, el portafolio docente $y$, por supuesto, el diario de un docente, es decir el diario personal del docente, para aquellos que llevan uno. Reiteramos: la escritura docente, una escritura profesional, no es y no puede ser sólo una escritura técnica, sino que tiene y debe tener aspectos y sustentos personales, ya que la vida profesional del docente no está separada de su vida personal, sino que constituyen una unidad: la vida del ser humano que es el docente.

Como un apoyo a un tipo de escritura técnica como lo es la escritura docente, podemos ver la propuesta de Julia Cameron (1999; 2016). Cameron propone practicar una escritura de autocuido como rutina habitual, diaria, para sustentar y nutrir la creatividad en la vida. Esta es una propuesta que contribuye al desarrollo de capacidades como integridad emocional, respeto, dignidad, reconocimiento y disposición al aprendizaje, las cuales forman parte de las ocho capacidades esenciales para la educación superior propuestas por Walker (citada en Boni, Lozano y Walker, 2010, pp. 126-127). Estas capacidades, a su vez, son esenciales para una práctica docente de calidad.
Así, para que las actividades de escritura docente se realicen con calidad, es necesario no sólo saber cómo hacerlas, sino también que tengan un sentido y un lugar (un espacio físico y tiempos específicos) para el docente como parte de su rutina de trabajo y, por consiguiente, que tengan un sentido $y$ un lugar en su vida. Es necesario trabajar con los docentes a través de procesos y desarrollar junto con ellos prácticas que les sirvan para crear significados y sentido en sus vidas, $y$, con este sustento emocional funcionando, asistirlos para que dominen sus actividades de escritura docente e innoven a través de ellas. La escritura de autocuido tiene un potencial enorme para contribuir al logro de estos objetivos, como lo prueban las décadas de estudio y resultados positivos de la escritura expresiva (Pennebaker \& Smyth, 2016)

En esta investigación, como herramientas para incentivar el desarrollo de la escritura de autocuido en las docentes participantes, se propusieron: la escritura del flujo de pensamiento o de conciencia (Cameron, 1999 y 2016; Goldberg, 2001), para aprender a permitir la expresión de los pensamientos propios y mostrar una forma de disolver los bloqueos creativos, y el diario (Lowe, Prout \& Murcia, 2013), para lograr, esencialmente, la claridad mental. Y como actividades de escritura docente se trabajó en la planificación didáctica por competencias (Tobón, 2005) para Educación Artística y se orientó e incentivó a las maestras para la elaboración de un portafolio docente (Flores, 2008; FEPADE, 2008). 
Luego, para apoyar el desarrollo de capacidades como conocimiento e imaginación, disposición al aprendizaje y razón práctica (Walker citada en Boni, Lozano y Walker, 2010, pp. 126-127), se trabajó en la motivación a la lectura, desde la teoría y las pautas expuestas en Zetino (2014).

Cantú \& Villanueva (2008) muestran que la lectura y la escritura de los docentes deben verse como dos caras de la misma moneda, como un binomio inseparable, y no como actividades que puedan realizarse una con independencia de la otra: la motivación, las actitudes y las habilidades en una condicionan estos aspectos en la otra, siendo mayor la influencia, mayor el potencial transformador, del binomio escritura-lectura.

Por último, desde nuestras propias experiencias docentes, entendemos que una reapropiación (una o apropiación por primera vez) de la lectura en docentes en servicio, es más factible a partir de una apropiación de la palabra, de la propia palabra y de la palabra del grupo, que de una actividad estructurada de un modo y con connotaciones más escolares: primero leer y después escribir, asociando esa escritura a lo leído, como una tarea. ${ }^{5}$ Así, apostamos por partir de la escritura, de una escritura más personal, para tener más posibilidades de resignificar, desde la satisfacción y la perspectiva que da esa experiencia, la lectura.

\section{Enfoques educativos}

El módulo de escritura y literatura de esta investigación tiene a su base teoría, práctica e investigación sobre escritura de autocuido, escritura creativa y motivación a la lectura en entornos escolares y no escolares. Esta fundamentación se adaptó a la formación docente desde el enfoque constructivista (Gamero y Mercadillo, 2011), el enfoque contemporáneo de la educación artística (Valle, 2016; Valle \& Zetino, 2019), el enfoque de capacidades (Boni, Lozano \& Walker, 2010), el paradigma biocéntrico (Pérez, 2018; Nadais \& Silva, 2019) y, a partir de este último, el enfoque educativo biocéntrico (Pérez, 2018; Nadais \& Silva, 2019), en los que se basa a su vez la investigación más amplia de la que este estudio forma parte.

\section{Capacidades}

Nussbaum (2012) define las capacidades como respuestas a la pregunta «¿Qué es capaz de hacer y de ser esta persona?» (p. 31); como «un conjunto de oportunidades (o libertades sustanciales)» que las personas pueden llevar a la práctica por elección propia (p. 29). Y precisa que las capacidades son objetivas y están determinadas por factores objetivos: «no son simples habilidades residentes en el interior de una persona, sino que incluyen también las libertades o las oportunidades creadas por la combinación entre esas facultades personales y el entor- 
no político, social y económico» (p. 32). «En general», dice la autora, «[...] el enfoque de las capacidades (al menos, en mi versión) se centra en la protección de ámbitos de libertad tan cruciales que su supresión hace que la vida no sea humanamente digna» (p. 43).

La investigación incluyó la promoción de capacidades por diversos motivos: por un lado, la búsqueda de estas a la educación artística contemporánea; luego, la búsqueda de un desarrollo humano integral del alumnado tiene como condición un desarrollo humano integral, en todo cuanto sea posible, del profesorado, y desde el enfoque de las capacidades, estas son parte esencial de dicho desarrollo; también, una formación integral no puede ser meramente procedimental, sino que debe incluir el desarrollo de los aspectos emocional y social de las personas, los cuales están contemplados como parte del enfoque de las capacidades de Nussbaum (2012); por último, la autonomía necesaria para la apropiación y puesta en práctica a largo plazo de las competencias docentes en educación artística contemporánea pasa necesariamente por una autonomía personal, que requiere de la posesión de determinadas capacidades. «Promover capacidades es promover áreas de libertad, lo que no es lo mismo que hacer que las personas funcionen en un determinado sentido», anota Nussbaum (2012, p. 36). Para este estudio también se tomó en cuenta la propuesta de capacidades para la educación superior de Boni, Lozano \& Walker (2010), que incluyen, entre otras, las capacidades de imaginación, autocuido, reflexión y la capacidad de aprender sin miedo.

\section{Metodología}

Basados en Chiva-Bartoll, Capella \& Pallarès (2018, pp. 279ss), realizamos el estudio en cuatro fases: 1. diagnóstico y reconocimiento de la situación inicial, 2. desarrollo de un plan de acción para mejorar las prácticas actuales, 3. actuación e investigación de los efectos en el contexto y 4. reflexión y bases para una nueva planificación. Relatamos dichas fases.

\section{Diagnóstico y reconocimiento de la situación inicial}

«El diagnóstico acarrea definir el problema a resolver y declarar las intenciones de mejora, para lo que es imprescindible describir el contexto y las diferentes perspectivas existentes», plantean Chiva-Bartoll, Capella \& Pallarès (2018, p. 280). El diagnóstico se realizó a partir de dos fuentes de información: los logros de los módulos anteriores y el inicio del módulo de literatura.

En los dos módulos que precedieron al de literatura se alcanzaron logros importantes con el grupo participante, como el desarrollo de la afectividad personal y grupal, la consolidación de una comunidad educativa, y una actualización teórica y una sensibilización sobre nuevos paradigmas de arte, educación y educación artística. Se debe mencionar que la sensibilización sobre estos últimos elementos se realizó a través de la metodología vivencial del enfoque educativo biocéntrico, que utilizó como herramienta principal la Vivencia. Ante los buenos resultados con dicha herramienta, esta se incorporó también a este módulo. 
Uno de los conceptos y recursos de la educación biocéntrica es la Vivencia ${ }^{6}$, «la herramienta metodológica de la Biodanza ${ }^{7}$, que incluye el encuentro de un grupo de personas, el movimiento y la música para evocar el concepto de estar "aquí y ahora"» (Valle \& Zetino, 2019, p. 20). Además, la Vivencia incluye las consignas de los ejercicios que la conforman; dichas consignas buscan ser no sólo instruccionales, sino también afectivas, para lo cual requieren ser elaboradas con recursos retóricos, lo que las vuelve poéticas.

Pérez (2018) explica el concepto de Vivencia:

La perspectiva de este enfoque [la educación biocéntrica] hace una inversión epistemológica; es decir, se conoce a partir de la emoción, a partir del diálogo emocionado, a partir de la vivencia y la afectividad[,] que van a nutrir el mundo del pensamiento racional[,] enraizándolo en la experiencia $[\ldots]$

La vivencia integradora es el centro organizador de la corporeidad y de la racionalidad. Cuando la persona se sienta a sí misma y se conecte [lo cual es promovido por medio de la Vivencia], aumentará la empatía, ampliará la percepción y la conciencia, la compasión y la comprensión. La cualidad de la inteligencia se organiza en función de su fuente afectiva. (p. 76)
De este modo, por medio de la Vivencia, la educación biocéntrica busca «educar en la inmanencia del cuerpo, desde la vivencia, la emoción, abriendo puertas a una mirada más totalizadora y abarcadora» (Pérez, 2018, p. 76). Y la autora cierra el apartado sobre la Vivencia planteando que «Se puede aprender y enseñar a amar, siendo el amor un centro generador de vida y centro del proceso educacional» (Ídem).

Se señala también que la Vivencia puede enmarcar experiencias de enseñanza aprendizaje, potenciándolas y profundizándolas. Pérez (2018) ha utilizado la Vivencia como parte de sus experiencias de formación docente y ha documentado aplicaciones de esta por parte de otros docentes en diversos países, incluidas experiencias de la Dra. Valle Contreras (en El Salvador y China), integrante de este equipo de investigación (Pérez, 2018, p. 380). Así, en este estudio, la Vivencia se ha utilizado para enmarcar experiencias de escritura y de lectura como parte de una formación docente continua en educación artística.

Se anota que la exploración de los potenciales del paradigma educativo biocéntrico ya fue planteada en el Programa de Estudios de Educación Artística del Programa Nacional de Formación Docente (2017-2019), por lo que posee un marco institucional que la sustenta en esta asignatura (MINED, 2017).

6 Con mayúscula, para distinguirla de la vivencia en sentido de experiencia.

7 Propuesta educativa y re-educativa creada por Rolando Toro Aráneda (Chile, 1924-2010), educador, psicólogo, antropólogo y poeta. La educación biocéntrica es una extensión de la biodanza, planteada también por Toro. Para ampliar, ver: Pérez (2018) y Valle \& Zetino (2019). 
Ahora, volviendo a los logros de la formación precedentes al módulo de literatura, el desarrollo de la afectividad y la consolidación de una comunidad de aprendizaje habían producido mejoras en el clima de la escuela: el clima laboral del equipo docente había mejorado, y los estudiantes, a partir de testimonios de las docentes, percibían un ambiente de mayor afectividad, y ellos mismos se expresaban más afectivamente. Estos son efectos personales y sociales de la investigación-acción que estaba en curso. Por último, las docentes habían realizado con los estudiantes algunas experiencias de educación artística con enfoque contemporáneo de modo no estructurado.

Sin embargo, también se detectó que las docentes se encontraban con dificultades para llevar a cabo diversas actividades: las lecturas asignadas, la escritura de un diario de la experiencia y la planificación didáctica por competencias que requiere la educación artística con enfoque contemporáneo. Ante estas dificultades, se diseñó el módulo de literatura para posibilitar una mirada nueva de estas actividades, favoreciendo su utilización como herramientas de desarrollo profesional y su realización efectiva.

Tal como las caracterizan Cantú \& Villanueva (2008), y lo pudimos comprobar en los dos módulos anteriores, las experiencias y prácticas de escritura y lectura que tenían las docentes eran negativas, en el caso de las experiencias, y fragmentarias, en el caso de las prácticas, volviéndose necesario atender estos aspectos para posibilitar su continuidad una vez que finalizara la formación docente.
Se buscó que las docentes experimentaran la escritura como medio de expresión de emociones y clarificación del pensamiento, y la lectura, por medio de la lectura de literatura, como una actividad motivadora, placentera; como texto artístico que promueve la imaginación, el asombro, la introspección y la reflexión.

Luego, al inicio del módulo de escritura, se les preguntó a las docentes sobre sus expectativas para el módulo: qué deseaban aprender en el módulo para ellas y para sus estudiantes. Las respuestas personales se dirigieron a cultivar más y mejor la lectura: «Tener más disciplina para leer», «Desarrollar más la comprensión lectora», «Me siento estancada en métodos de lectura»; y las respuestas sobre el trabajo con los estudiantes se refirieron a la motivación para leer: «Motivar a mis estudiantes, para que tengan una mejor expresión», «Motivar a mis estudiantes porque yo lo transmita», «Encontrar estrategias para motivar a la lectura». Es decir: coincidieron en los deseos de tener buenas experiencias de lectura y de motivar a la lectura.

Y como último elemento del diagnóstico, entra un hecho no previsto. Para el final del módulo II, la planta docente, en una reunión realizada por iniciativa propia, había acordado crear condiciones para el desarrollo de experiencias de educación artística con el alumnado: modificar horarios y asignar al personal de apoyo de la escuela para asistir a las maestras en la asignatura. Sin embargo, dos meses después, al iniciar el módulo de literatura, aún no se había comenzado a 
implementar la asignatura con el enfoque contemporáneo. Al preguntar a las maestras por las dificultades para implementar el enfoque, estas expresaron que aún tenían grandes dudas. La directora del centro escolar manifestó que el aprendizaje del enfoque era un proceso, para hacer ver que todavía no había llegado a un manejo de este. Ella, que también enseña en parvularia, expresó: «Ha habido cierto temor a equivocarnos, a no lograr los objetivos esperados. [...] Estamos convencidas de eso [llevar a la práctica el enfoque contemporáneo de la educación artística], pero hay ciertas inseguridades» (módulo III, taller 2). Su opinión resumía la de las demás maestras.

De este modo, la situación inicial de la investigación estaba conformada por logros de las dos etapas anteriores y, en gran medida, por el desafío de concretar la escritura docente en la práctica de la asignatura.

\section{Desarrollo de un plan de acción para mejorar las prácticas actuales}

Ante la necesidad expresada en el apartado anterior, el equipo evaluó el rumbo a seguir durante el tercer módulo, y el plan inicial del módulo se modificó para atender dicha necesidad y, al mismo tiempo, tratar de desarrollar las capacidades y competencias proyectadas inicialmente. Así, se les propuso a las docentes realizar un taller de planificación didáctica por competencias, para asistirlas paso a paso en la planificación de la asignatura desde el enfoque contemporáneo de la educación artística, lo cual aceptaron con gusto. Dicho taller abarcó cuatro de las nueve sesiones del módulo (talleres 3 a 6), con lo cual modificó su estructura y lo dirigió a atender de un modo más específico necesidades urgentes del equipo docente. El módulo original y el módulo final pueden visualizarse en la siguiente tabla: 


\section{Tabla 1. Módulo original y modificación para atender necesidades de docentes}

\begin{tabular}{|c|c|}
\hline Plan del módulo original & Talleres del módulo modificado \\
\hline $\begin{array}{l}\text { 1. El universo comienza en nosotros: escribir nues- } \\
\text { tros sueños. (Contacto con los valores personales, } \\
\text { utopías y proyectos). }\end{array}$ & $\begin{array}{l}\text { 1. El universo comienza en nosotros: escribir nues- } \\
\text { tros sueños }\end{array}$ \\
\hline $\begin{array}{l}\text { 2. Escribir para aprender y transformar: el portafolio } \\
\text { y el proyecto }\end{array}$ & $\begin{array}{l}\text { 2. Redescubrir el placer de leer. (Motivación a la } \\
\text { lectura) }\end{array}$ \\
\hline 3. TICs: Herramientas básicas de escritura & $\begin{array}{l}\text { 3. Taller de diseño de experiencias de enseñanza } \\
\text { aprendizaje en Educación Artística (I): Introducción } \\
\text { al enfoque por competencias }\end{array}$ \\
\hline $\begin{array}{l}\text { 4. Una caja de herramientas: recursos retóricos } \\
\text { básicos }\end{array}$ & $\begin{array}{l}\text { 4. Taller de diseño de experiencias de enseñanza } \\
\text { aprendizaje en Educación Artística (II): Planificación } \\
\text { didáctica de una sesión de Educación Artística por } \\
\text { competencias }\end{array}$ \\
\hline 5. La poesía: una música universal & $\begin{array}{l}\text { 5. Taller de diseño de experiencias de enseñanza } \\
\text { aprendizaje en Educación Artística (III): Planificación } \\
\text { didáctica de una sesión de Educación Artística por } \\
\text { competencias }\end{array}$ \\
\hline 6. Narrativa: el poder de las historias & $\begin{array}{l}\text { 6. Taller de diseño de experiencias de enseñanza } \\
\text { aprendizaje en Educación Artística (IV): Práctica de } \\
\text { sesión diseñada por el grupo }\end{array}$ \\
\hline $\begin{array}{l}\text { 7. ¿Qué leemos? Diseñar un canon de lectura para } \\
\text { mis alumnos }\end{array}$ & $\begin{array}{l}\text { 7. La poesía, una música universal (I): La metáfora, } \\
\text { figura y experiencia }\end{array}$ \\
\hline $\begin{array}{l}\text { 8. Comunicarme con mi comunidad: proyectos de } \\
\text { educación artística en la escuela potenciados por } \\
\text { la escritura }\end{array}$ & $\begin{array}{l}\text { 8. La poesía, una música universal (II): Hagamos } \\
\text { acción la poesía. (Planificación didáctica) }\end{array}$ \\
\hline $\begin{array}{l}\text { 9. Cierre de proyectos transversales: proyectos de } \\
\text { CE y portafolio de experiencias docentes }\end{array}$ & $\begin{array}{l}\text { 9. Creación colectiva para la Educación Artística: La } \\
\text { poesía como estrategia creativa }\end{array}$ \\
\hline
\end{tabular}

Fuente: Elaboración propia.

Perlo \& Sagastizabal (2002) señalan que «La realidad investigada puede mostrarnos, durante el trabajo de campo, variables o factores no contemplados en el abordaje inicial o nuevos enfoques y elementos que ampliarán y modificarán la perspectiva inicial $[\ldots] \gg(\mathrm{p}$.
104). De este modo, el módulo de escritura y literatura se rediseñó para alcanzar un doble resultado: apoyar el aspecto actitudinal de la práctica docente, es decir dar herramientas que hicieran posibles la motivación, la resiliencia y la creación de sentido cons- 
tantes necesarias para realizar una buena práctica docente; y apoyar actividades de escritura docente que requiere la educación artística con enfoque contemporáneo: la investigación, tanto artística como educativa; la planificación didáctica por competencias, y el registro de las experiencias de enseñanza aprendizaje (MINED, 2017, pp. 8-14). Como puede verse en la tabla, el plan original del módulo, en la práctica, se orientó hacia actividades y áreas específicas, siendo las principales la planificación por competencias y la experiencia de la poesía, y hacia su profundización.

\section{Actuación e investigación de los efectos en el contexto}

Se desarrolló el módulo de formación docente continua con las participantes ya mencionadas: la planta docente del centro escolar (5 personas) y participantes de la ONG que apoya a la escuela (4 personas). La investigación se desarrolló a través del método de la observación participante, alternándose los investigadores, que fueron los facilitadores de la formación, en el rol de participantes de la formación, para poder disponer de esta perspectiva. Además, la observación se limitó a los momentos de la formación, realizada en las instalaciones de la Universidad Dr. José Matías Delgado, pues por motivos de seguridad no se pudo asistir a prácticas regulares de las docentes en su centro escolar. De este modo, los efectos de la formación en el contexto del centro escolar se conocen por los registros y testimonios de las participantes.
Para las participantes, se plantearon dos modos de registro: un diario de la experiencia, para ser escrito libremente (se anota que este diario ya venía siendo utilizado desde los módulos anteriores) y un portafolio docente, para registrar las experiencias de enseñanza aprendizaje en educación artística realizadas con los estudiantes. Además, el equipo de investigación elaboró un informe escrito de cada taller, y se creó un amplio registro de archivos de audio, fotografías y videos de los diversos momentos de los talleres.

Los talleres tuvieron tres tipos de momentos: dialógicos, teóricos y prácticos. Cada taller iniciaba con un diálogo de los investigadores con las participantes acerca de cómo estaban, sus actividades desde el último taller, y los aprendizajes, hallazgos y aplicaciones que habían hecho a partir de lo trabajado en el taller anterior. Luego se podía pasar a un momento teórico, para mostrar los fundamentos y procedimientos de las actividades a realizar en el taller. Y por último, el momento práctico se realizaba enmarcado por la Vivencia como herramienta metodológica para enmarcar y potenciar los aprendizajes.

La forma más básica de utilizar la Vivencia fue abrir y cerrar los talleres de discusión teórica y escritura técnica con rondas, según la metodología de la biodanza, como una forma de crear un clima de aprendizaje afectivo y comunitario. Una segunda modalidad fue enmarcar las experiencias de escritura y lectura con la secuencia de ejercicios de biodanza que constituyen la Vivencia, lo 
cual propició espontaneidad, fluidez y altos grados de creatividad en las participantes. Esto queda ejemplificado en la aplicación más compleja que se hizo de la Vivencia, que fue la creación colectiva y puesta en práctica de una Vivencia como ejercicio de planificación didáctica. La actividad curricular que fue enmarcada por la Vivencia fue la escritura de un poema. El resultado fue una secuencia de ejercicios muy fluida, y la escritura de un poema en un estado mental muy sensible y creativo, sin juicios hacia la propia escritura, sino por el contrario: con gran apertura hacia la expresión propia y de los demás. Un ejemplo de la calidad de pensamiento artístico potenciada en esta experiencia es este fragmento de un poema de una de las participantes:

\section{La vida es eso,}

eso que encontramos en nuestro interior.

Una música, una mirada, un abrazo.

Es darme cuenta de que estoy aquí viva.

Sobre la observación de los efectos en el contexto, el contexto inmediato del que debemos hablar es el equipo docente del centro escolar. Pudimos observar, específicamente en este módulo, y de manera general a lo largo de la formación docente en su totalidad, una mejora progresiva del clima afectivo del equipo docente, la cual las docentes también percibieron y refirieron, agregando que la vivencia del equipo docente se había transmitido a los estudiantes. Diversos testimonios y registros de las maestras mostraron los cambios actitudinales de los estudiantes, derivados, en primer lugar, de los cambios actitudinales de las maestras, y luego, de la incorporación a la práctica docente de elementos de la propuesta formativa.

\section{Reflexión y bases para una nueva planificación}

Como se muestra en el apartado de resultados, el módulo promovió capacidades personales y grupales en las participantes, tales como el desarrollo de la sensibilidad estética, la imaginación, la conciencia de la propia capacidad poética y el sentimiento de comunidad. Asimismo, se desarrolló una práctica asistida de planificación didáctica por competencias para educación artística, lo cual provocó un conflicto cognitivo en el equipo docente acerca de la planificación tradicional (por objetivos) y la planificación con enfoque en competencias. Sin embargo, no se logró la incorporación inmediata de este último enfoque educativo en la práctica de las docentes.

Este hecho hace que el equipo de investigación vea la necesidad de plantear la planificación por competencias de un modo distinto en futuras implementaciones de la formación docente desarrollada, contemplando entre las posibilidades un módulo exclusivo para la planificación y la práctica.

\section{Resultados}

Al cierre del módulo, se pudo observar en las participantes, y estas dieron referencia de esto, de marcos vivenciales, de sensibilización y afectivos sobre la experiencia literaria, en particular sobre la comprensión de 
la poesía y la creación de esta, siendo los hechos más destacables el permitirse escribir los propios pensamientos sin juzgarlos y la confianza en la propia capacidad para escribir utilizando recursos literarios. Además, se registró un desarrollo básico de competencias técnicas de escritura, específicamente para la planificación didáctica con enfoque en competencias.

Entre los marcos de sensibilización desarrollados, se encuentran una experiencia de lectura por placer y una ampliación teórica y vivencial sobre la poesía y, en concreto, sobre la metáfora. Acerca de dicha experiencia, la directora del centro escolar expresó en la sesión final del módulo:

[La poesía] se puede experimentar de muchas formas, yo creo $[\ldots] .[\ldots]$ yo lo expreso así: el encontrar una serie de conexiones. [...] hoy, por ejemplo, [en la Vivencia] he experimentado la poesía sin palabras, la poesía muda. Y la he sentido, y la he disfrutado al máximo [...]. [...] el sentir, yo creo que muchas veces ni siquiera lo podemos explicar, ¿verdad? Simplemente lo vivimos, lo experimentamos [...] Yo, por ejemplo, de hoy me llevo una gran experiencia: de venir de un estado de ánimo y me voy a ir de otro, porque... creo que logré conectar con todos, y creo que la mayoría también conectó conmigo. [...] [La poesía] es eso, yo creo: es encontrar la esencia de las cosas, a veces en lo más simple, en la simplicidad; a veces [...] en lo insignificante, y que se vuelve tan valioso. (Taller 9).
Además, las prácticas de escritura de autocuido fueron llevadas a cabo esporádicamente, reportando las maestras beneficios como la claridad mental al organizar ideas. Algo parecido sucedió con la lectura por placer: fue desarrollada al ritmo propio de cada participante (lo cual implicó incluso la devolución de los libros leídos hasta el módulo siguiente de la formación); y sus evidencias consistieron en pláticas, que fueron entusiastas, sobre las lecturas realizadas. En el marco de los Derechos del Lector propuestos por Pennac (1992), esto es positivo, y preferible a no tener comentarios sobre lo leído. Se consigna que no se hizo préstamo de nuevos libros, quedando la lectura de un libro por elección propia como una única experiencia.

Acerca de las competencias de escritura, durante los talleres sobre planificación didáctica, las docentes, asistidas por el equipo de investigación, trabajaron en la elaboración de una carta didáctica por competencias para Educación Artística, siendo esta una experiencia que se dio dentro del espacio de la formación, pero que no fue continuada en el centro escolar. Las docentes señalaron que les estaba llevando tiempo asimilar un nuevo modelo de planificación. Así, durante el transcurso del módulo, no se logró instalar una rutina de planificación didáctica para Educación Artística ni individual ni grupalmente. Por un lado, esto podría explicarse desde la curva de aprendizaje que implica todo procedimiento nuevo; en el caso de competencias docentes, estudios muestras que la apropiación de estas se hace afectiva a mediano plazo. (Ver, por ejemplo, el desarrollo de competencias digitales por docen- 
tes, en Cabero \& Martínez, 2019). Por otro lado, las condiciones objetivas de las maestras tendrían un peso mucho mayor del que se dimensionaba al plantear obstáculos para instalar una nueva rutina de planificación.

Se anota que una miembro de la ONG de apoyo planificó por competencias una sesión de biodanza para una capacitación que impartió como consultora, evidenciando su comprensión del enfoque y abriendo una oportunidad de apoyar a las maestras en este aspecto luego de la formación.

Luego, sobre el portafolio docente, dos de las maestras presentaron los suyos, aunque no durante el módulo, sino en el módulo siguiente de la formación, mostrando así el registro de algunas experiencias de enseñanza aprendizaje con sus estudiantes.

Por último, sobre el diario de la experiencia, se compartieron también algunas anotaciones, y se comprobó la preferencia por el portafolio como registro de la puesta en práctica del enfoque.

Ahora, hay otros aspectos en los que deben señalarse logros importantes, como lo son el desarrollo de la afectividad a nivel personal y grupal y la mejora de la convivencia en el equipo docente. Tanto durante este módulo como durante el resto de la formación docente, las participantes encontraron en la formación un espacio de autocuido y creación de comunidad educativa, lo cual tuvo en efecto en su práctica docente y posibilitó aprendizajes que podrán ser reelaborados y puestos en práctica luego de que la formación finalice.
La utilización de la Vivencia como un recurso educativo probó ser efectiva para el desarrollo duradero de estas dimensiones.

De este modo, si bien las herramientas técnicas que el módulo propuso no se instalaron como rutina en la práctica de las docentes, estas, y el contexto en el que se aprendieron, posibilitaron una visión ampliada de las mismas: «[La escritura y la literatura] son herramientas o recursos integradores en nuestra personalidad, para ser seres humanos más sensibles, compasivos y solidarios. Es una herramienta educativa, pero sobre todo de crecimiento y salud mental, emocional y física», expresó una de las maestras en la evaluación del módulo.

Además, las recomendaciones que las maestras dieron para futuras realizaciones del módulo, se dirigen hacia aspectos básicos de la formación docente en general: «Fortalecer más el hábito de la lectura a nivel personal»; «[Dar] más tiempo para no correr [...]»; «Que se dé continuidad al proceso», «Mantener el seguimiento al grupo participante».

Estas recomendaciones son muy significativas, pues se enfocan en aspectos del proceso de formación docente previos o necesarios para las actividades de la práctica docente, y no en las actividades técnicas en sí mismas.

\section{Conclusiones}

La investigación desarrollada proporciona información relevante para la mejora de la formación docente continua en educación artística. 
Sobre el objetivo de la investigación, concluimos que la experiencia literaria, una experiencia integral, vivencial, de la escritura y de la lectura, tiene una incidencia favorable en la promoción de capacidades y competencias en docentes de educación artística en formación continua. De este modo, a partir de los resultados del módulo de literatura que se desarrolló, se concluye que una visión renovada y experiencial de dichos campos, como una parte habitual y satisfactoria de la vida, y no como obligación escolar o laboral, incide positivamente en la práctica docente en general, al generar estados de bienestar y propiciar el empoderamiento, tanto individual como colectivo en ambos casos. En estos resultados, se señala que el desarrollo de capacidades, tales como la sensibilidad poética, la creatividad y la afectividad, fue una condición para el desarrollo de competencias. Es decir que la atención a la dimensión personal de las docentes fue indispensable para obtener resultados en el aspecto técnico de la formación.

De este modo, concluimos que el aspecto afectivo en la formación docente continua no es un extra, no es algo opcional; es parte de la creación de nuestro clima de trabajo. Evaluamos que realizar una sesión de trabajo que no hubiera tocado las sensibilidades de las docentes, que hubiera sido puramente técnico-instrumental, se habría quedado descontextualizado, pues ese fue el centro de nuestra propuesta pedagógica, inspirada en el paradigma biocéntrico (Pérez, 2018) y en una línea del arte contemporáneo (Alberro, 2011, p. 166). Ese tipo de capacidad afectiva es la que el equipo buscaba dejar instalada en la planta docente como comunidad de aprendizaje, ya que de ese modo también crece en su potencial creativo, innovador, y como mediadora efectiva y afectiva, generadora de vitalidad, para el alumnado del centro escolar.

Dicho desarrollo de capacidades afectivas y para la convivencia durante el módulo de formación docente continua, así como también durante los módulos precedentes, demuestra el potencial del enfoque educativo biocéntrico para propiciar cambios en estas dimensiones a nivel individual y grupal. Ya que esto ha podido lograrse en formación docente continua, se recomienda iniciar experiencias para incorporar el paradigma biocéntrico a la formación docente inicial en Educación Artística, y a la formación docente inicial en general. De manera particular, se comprobó la efectividad de la Vivencia como herramienta metodológica para enmarcar experiencias de aprendizaje sobre escritura, en este caso específico, sobre creación poética.

Sobre la escritura docente en la formación continua, debe investigarse más sobre cómo orientar su práctica. Ante el bajo dominio del equipo docente de la planificación con enfoque en competencias, el equipo considera que fue valioso tomar el tiempo necesario para trabajar con el equipo en dicho enfoque, para mejorar la comprensión de sus conceptos básicos. Es necesario desarrollar procesos de formación extensos con los docentes, y conocer y comprender los procesos de aprendizaje. Como se mencionó en los resultados, existe una curva de aprendizaje, y 
una apropiación de competencias docentes se iría haciendo efectiva en un mediano plazo. (Ver, Cabero \& Martínez, 2019).

Además, se concluye que en la formación docente continua es necesario trabajar con base en las necesidades de los docentes y diseñar procesos de formación flexibles y abiertos, para que de este modo sea posible el desarrollo significativo de competencias pedagógicas. En esta investigación entendemos por flexibilidad la capacidad para observar y escuchar el proceso, de dejar que el proceso de formación y los procesos de la comunidad educativa hablen, para que cada etapa del trabajo responda principalmente a las necesidades de ese momento, en vez de ceñirse mecánicamente al plan del módulo o a ideas previas o no examinadas. A partir de nuestra experiencia, consideramos que una formación docente continua funcional debe ser flexible, y así tener la capacidad de modificar su plan original para atender las necesidades de los docentes a quienes se está formando.

También, se debe trabajar sistemáticamente para propiciar la creación de rutinas de lectura por placer y de escritura creativa y de autocuido en la vida de los docentes, tanto en la formación inicial como en la continua.
Acerca de las dificultades encontradas durante el módulo para el desarrollo de competencias de escritura docente, se hace la observación de que estas también están estrechamente relacionadas a las condiciones objetivas de vida y de trabajo de los docentes, las cuales son, en El Salvador, en general, difíciles, adversas.

Finalmente, en la dimensión personal, hacemos la observación de que realizar esta formación docente en las instalaciones de la universidad influyó positivamente en la formación, pues el proceso se desarrolló en un entorno académico, y esto hizo sentir a las maestras como profesionales, les devolvió esta dimensión de sí mismas. El equipo de investigación observa que la dimensión profesional se diluye o se pierde en el día a día de las maestras, pues su entorno hace que deban funcionar como madres, al tener que dar a los niños un apoyo emocional que estos muy probablemente sólo encuentran en la escuela. En este sentido, es vital orientar a los docentes para trabajar la parte afectiva en la escuela sin que sea desgastante, mostrar cómo canalizar la afectividad y potenciarla en el proceso educativo. 
AKADEMOS Julio-Diciembre 2020, n. ${ }^{\circ} 35$, ISSN: 1995-4743

\section{Referencias}

1 Alberro, A. Periodizar el arte contemporáneo. En: Alberro, A. (Coord.). (2011). ¿Qué es arte contemporáneo hoy? (Simposio internacional). Navarra: Universidad Pública de Navarra. Págs. 155-169.

2 Boni Aristizábal, A; Lozano Aguilar, J. F. \& Walker, M. (2010). La educación superior desde el enfoque de capacidades. Una propuesta para el debate. REIFOP, 13(3), 123-131. https://dialnet.unirioja.es/servlet/ articulo? codigo $=3307562$

3 Blanchard Giménez, M. (coord.). (2014). Transformando la sociedad desde las aulas: Metodología de Aprendizaje por Proyectos para la Innovación educativa en EI Salvador. Madrid: Narcea.

4 Cabero Almenara, J. y Martínez Gimeno, A. (2019). Las tecnologías de la información y comunicación y la formación inicial de los docentes: modelos y competencias digitales. Profesorado: Revista de Currículum y Formación de Profesorado, 23(3), 247-268. https://hdl. handle.net/11441/89544

5 Cameron, J. (1999). The Right to Write. Los Angeles: Tarcher/Penguin.

6 Cameron, J. (2016). El camino del artista. Ciudad de México: Aguilar.

7 Cantú Valadez, M. \& Villanueva Gutiérrez, O. (2008). Prácticas de lectura, escritura y el triple presente de la formación docente. Red, 5, 28-43. https:// www.academia.edu/15620879/Pr\%C3\%A1cticas_ de_lectura_escritura_y_el_triple_presente_de_la_ formaci\%C3\%B3n_docente

8 Carlino, P. \& Martínez. S. (Coord.). (2009). La lectura y la escritura: un asunto de todos/as. Argentina: Universidad Nacional de Comahue. (Libro en PDF). http://rdi. uncoma.edu.ar:8080/handle/123456789/1575

9 Chiva-Bartoll, Ò., Capella Peris, C., \& Pallarès Piquer, M. (2018). Investigación-acción sobre un programa de aprendizaje-servicio en la didáctica de la educación física. Revista De Investigación Educativa, 36(1), 277293. https://doi.org/10.6018/rie.36.1.270581
10 DeSalvo, L. (2000). Writing as a Way of Healing. How Telling our Stories Transforms our Lives. Boston: Beacon Press.

11 FEPADE. (Entrevistador). (2008). El portafolio docente. Una estrategia para apoyar la reflexión y el cambio. Revista Pedagógica AB-sé FEPADE, 10(1), 15-20.

12 Flores, I. (2008). ¿Qué aprendemos de lo que vemos y escuchamos? El docente como profesional reflexivo. Revista Pedagógica AB-sé FEPADE, 10(1), 8-14.

13 Gamero Flores, D. del C. \& Mercadillo de Monterrosa, A. D. (2011). Manual del docente universitario. Antiguo Cuscatlán, El Salvador: Editorial Delgado.

14 Goldberg, N. La escritura, una terapia creativa. Barcelona, Oniro, 2001.

15 Labarthe, J. T. \& Herrera Vásquez, L. (2016). Potenciando la creatividad humana: Taller de escritura creativa. Papeles de trabajo, 31. http://hdl.handle. net/2133/12783

16 Lowe, G. M.; Prout, P. \& Murcia, K. (2013). I See, I Think I Wonder: An Evaluation of Journaling as a Critical Reflective Practice Tool for Aiding Teachers in Challenging or Confronting Contexts. Australian Journal of Teacher Education, 38(6), 1-16. En internet: http:// ro.ecu.edu.au/ajte/vol38/iss6/1

17 Martín-Baró, I. (2010). La investigación y el cambio social. Revista Salvadoreña de Psicología, 2(1), pp. 91-98. (Ponencia presentada en 1987). http:// www.uca.edu.sv/coleccion-digital-IMB/wp-content/ uploads/2015/11/2010-La-investigaci\%C3\%B3n-y-elcambio-social-RP2010-1-2-91_98.pdf

18 Martínez, M. C. (2010). Docentes de educación artística. Experiencias en el marco de la formación continua. Revista Iberoamericana de Educación, 52, 95-108.

MINED. (2017). Plan de Estudios de Educación Artística. (Programa de Formación de Docentes en Servicio del Sector Público del Ministerio de Educación). En internet: http://minedupedia.mined.gob.sv/lib/exe/fetch. php?media=files:plan_educarte_2017.pdf 
20 Nadais, C. \& Silva, A. M. (2019). Biocentric Education: From Rolando Toro Model to the Paradigm. (Comunicación científica). (PIXEL. International Conference The Future of Education, edition 9). https://conference.pixel-online.net/FOE/files/foe/ed0009/FP/5950ESI4080-FP-FOE9.pdf

21 Neira Leguizamón, J. V. (2017). La escritura creativa como contribución a la experiencia estética. (Tesis de Licenciatura en Español e Inglés). Bogotá: Universidad Pedagógica Nacional. URI: http://hdl.handle. net/20.500.12209/9226

22 Nussbaum, M. C. (2012). Crear capacidades. Propuesta para el desarrollo humano. Barcelona: Paidós.

23 Ospina, V. (2016). El docente del nivel inicial: retos para la formación profesional y continua. En Revista Educación y Humanismo, 18(30), 107-122. http:// dx.doi.org/10.17081/eduhum.18.30.1325

24 Pennac, D. (1992). Como una novela. Barcelona: Anagrama.

25 Pennebaker, J. W. \& Smyth, J. M. (2016). Opening Up by Writing It Down. How Expressive Writing Improves Health and Eases Emotional Pain. (3rd Ed.). New York: The Gilford Press. (Ebook).

26 Pérez, N. (2018). Educación y Cultura como Centros de Vida. Una pedagogía del afecto y la creatividad para docentes y familia. Resistencia, Argentina: ConTexto Libros.

27 Perlo, C. L. \& Sagastizabal, M. A. (2002). La investigación acción como estrategia de cambio en las organizaciones. Cómo investigar en las instituciones educativas. ( $2^{\mathrm{a}} \mathrm{Ed}$.). Buenos Aires: Editorial Stella/ La Crujía Ediciones.

28 Suárez, V. (2014). La lectura como experiencia estético-literaria. Enunciación, 19(2), 215-227. http://dx.doi. org/10.14483/udistrital.jour.enunc.2014.2.a03 petencias: Pensamiento complejo, diseño curricular y didáctica. 2a Ed. Bogotá: Ecoe.

30 Valle Contreras, M. E. (2011). La Educación Artística en la enseñanza básica en El Salvador. Antiguo Cuscatlán: Centro de Investigaciones en Ciencias y Humanidades $(\mathrm{ClCH})$, Universidad Doctor José Matías Delgado.

31 Valle Contreras, M. E. (2016). Pensar la educación artística en el currículo nacional: evidencias, reflexiones para imaginar un nuevo escenario de la asignatura en El Salvador. Revista de Humanidades y Ciencias Sociales (CENISCH), n. ${ }^{\circ}$, II época, 15-49. https://doi. org/10.5377/rhcs.v0i8.6628

32 Valle Contreras, M. E.; Boulogne, S. M. \& Zetino, M. (2017). La calidad de la Educación Artística en la formación docente. Recorridos y desafíos. (Conversatorio).Akademos, 11(2), 87-100. https://doi.org/10.5377/ akademos.v2i29.6323

33 Valle Contreras, M. E. \& Zetino, M. (2018). Aportes de la Biodanza a la estética contemporánea: estética vivencial, creatividad existencial y mirada. Akademos, 12(2), 9-34. DOI: https://doi.org/10.5377/akademos. v2i31.8184

34 Zetino, M. (2014). Por qué no leemos. Factores de desanimación a la lectura y propuestas para la animación. Akademos, 8(3), 9-17. https://www.academia. edu/40263043/Por_que_no_leemos._Factores_de_ desanimaci\%C3\%B3n_a_la_lectura_y_propuestas_ para_la_animaci\%C3\%B3n

35 Zubiri, H. (2014). Escritura creativa para el empoderamiento en el aula. Textos de Didáctica de la Lengua y de la Literatura, 66, 61-70. https://www.grao. com/es/producto/escritura-creativa-para-el-empoderamiento-en-el-aula-tx06623403 
